\title{
The interplay between the physical internet and logistics: A literature review and future research directions
}

\author{
Safwen Ben Neila ${ }^{1}$, Abderahman Rejeb ${ }^{1, *}$, Péter Németh $^{1}$ \\ ${ }^{1}$ Széchenyi István University, Department of Logistics and Forwarding, \\ Egyetem tér 1. 9026 Győr, Hungary \\ *e-mail: abderrahmen.rejeb@gmail.com
}

Submitted:07/07/2021 Accepted:09/08/2021 Published online: 16/09/2021

Abstract: In recent years, there is a need for new methods and frameworks for planning transport systems, improving their efficiency, and addressing globalisation and sustainability challenges. In addition, the use of existing capacities and infrastructure has raised significant issues in the transport sector. To achieve an economically, environmentally, and socially sustainable logistics ecosystem, Benoit Montreuil introduces the concept of "Physical Internet" (PI) to increase the efficiency and effectiveness of logistics networks. As a ground-breaking transportation philosophy, the PI aims to revolutionise freight and logistics transport. Moreover, the PI can enhance logistics productivity through the organisation of large-scale pooling. Similar to Digital Internet that conveys data, the PI strives to connect, synchronise, and ship regular modular containers from the point of origin to an exact destination, thereby creating robust and collaborative logistics networks. While the literature on the PI is relatively growing, there is still a lack of reviews that synthesise this knowledge body, identify current trends and gaps, and advance the research more broadly. Therefore, this study aims to investigate the potentials of the PI for the development of sustainable logistics networks. Overall, 59 studies are selected from leading academic databases and further analysed. The review findings reveal that most scholars focus on the optimisation of transport at the tactical and organisational stage while devoting little attention to the contribution of the PI to the social sustainability of logistics compared to the economic and environmental aspects.

Keywords: physical internet (PI); logistics; transportation; sustainability

\section{INTRODUCTION}

Over the last decade, the fight against climate change has become one of the controversial topics and is currently identified as one of the severe problems societies have ever faced [1]. The main reason for climate change is attributed to Greenhouse Gases (GHG) emission and improper human activities. With the business and logistics community's mounting attention to this matter, there is a need for business talent, investment, problemsolving skills, and global reach to address the intractable problem of climate change and the inequality to equip workers for a new age of work [2]. Similarly, new management approaches are necessary to reduce socioeconomic and ecological imbalances, prevent economic crises, and achieve sustainability [3]. In response to increasing globalisation and customer requirements, the focus of organisations has shifted to developing optimisation initiatives that reduce logistics and transport costs [4]. One way to achieve this objective is to integrate the Physical Internet (PI) in the logistics sector. According to [5], the PI contributes to enabling order-of-magnitude improvements in logistics efficiency and sustainability. Thanks to its interconnections and open characteristics, the PI has the potential to help organisations meet sustainability challenges [6]. The approach of PI is based on establishing global cooperation and interoperability to facilitate both vertical and horizontal integration of transport systems globally and streamline sharing of resources, such as vehicles, data, spaces and warehouses [7]. Several scholars have started to consider the PI and scrutinise its possibilities in multiple stages of transport network planning.

During the realisation of the PI, strategic-level decisions include the design of distribution centres and the determination of the number of dock doors, flow racks, the material handling equipment used, and the travel distances. Whereas tactical-level 
decisions include the number of inbound trailers received, the number of unloaders and hauliers, the storage location assignment, etc. Operational-level decisions are concerned with inbound intralogistics, including unloaders' assignment and scheduling, loads-to flow rack assignment, and assignment and hauliers' scheduling [8]. Within the PI, the working principles and basics of the Digital Internet metaphorically correspond to handling, transportation, distribution, and storage of physical items. Breaking up the conventional business models, the PI creates a new setting of decentralised, collaborative, and cooperative logistics, spanning organisations, industries, and inter-organisational networks [9].

While the PI is receiving significant attention from researchers, there is still a lack of studies discussing the concept from the decision-making perspective. For example, [10] examine the state-of-the-art of the PI and provide several comprehensive future research directions. [11] discuss the synchromodal and PI state-of-the-art models and their architectures, methodologies, and observations in the academic literature. The authors' main goal is to analyse and investigate the connections between these synchromodal and PI models and explain how they are mutually reinforcing. Furthermore, [12] propose a PI structure recognising seven PI themes that can inform scholars and practitioners on the enablers, barriers, and policies of PI policies. Lastly, [13] study the PI literature using a qualitative content analysis method.

Unlike previous studies, we synthesise extant research on the PI using a coding framework consisting of three axes; the PI themes, the decision stages, and the sustainability aspects. The review contributes significantly to the literature because it increases researchers' understanding of the PI, the themes discussed in this literature, and the impact of this novel paradigm on sustainability. The systematic literature review includes reviews, articles and conference proceedings, business studies, and white papers. In addition, we carry out a descriptive and content analysis to have an overview of the PI literature's history and the themes discussed.

The structure of the paper is as follows. Section 2 presents a brief overview of the PI concept. Section 3 describes the methodology used for the review and content analysis. Section 4 provides an in-depth discussion of the results and findings. In the subsequent section, several recommendations for future research are highlighted, followed by a brief conclusion.

\section{OVERVIEW OF THE PI}

The PI is a metaphor of the Digital Internet applied to logistics networks and their services. The purpose behind the PI is to link up delivery networks, storage centres, vendors in an open global logistics system founded on physical, digital, and operational interconnectivity through encapsulation interfaces and protocols [5]. It is a perpetually evolving system driven by technological, infrastructural and business innovation [14]. Today, several players in the logistics industry are operating in isolation. Companies are autonomous and have their networks. However, with the support of the PI, the stakeholders of the logistics industry can maintain a high level of interoperability.

The PI provides an optimal solution on a broad scale, streamlining the logistics system, standardisation of resources (hardware and software), and the pooling of means. Through a standardised set of collaboration protocols, modular containers and smart interfaces, it is possible to develop a global logistics system and support the interconnection of logistics networks that can increase efficiency and sustainability [15]. Like the flow of data packages through the Internet, the PI is based on the use and handling of standardised smart boxes. These boxes can be visualised easily when moving throughout the supply chain, making logistics more efficient and sustainable. [5] argues that the way physical objects are handled, moved, stored, realised, supplied and used in the PI. He also affirms that it is a revolutionary concept, which is strikingly different from the currently prevailing theory and practice of transport, logistics and the supply chain. The PI takes advantage of the Digital Internet metaphor to shape the physical world and ensure global logistics efficiency and sustainability.

\section{RESEARCH METHODOLOGY}

In general, literature reviews aim to map, consolidate, and analyse a particular research area and find gaps to fill in to expand further the current information base [16]. Given that the PI literature exists in different outlets and spans several fields such as logistics, supply chain, and business, a comprehensive analysis of the current state of the PI is urgently needed. This research will provide better conceptualisation by updating the standard terminology of the PI and suggesting recommendations for future PI studies. As part of this focus, we carried out a systematic literature review to provide a rigorous and detailed analysis of the current research on the PI. Five steps were followed to ensure the extraction of suitable studies and their systematic inspection. Firstly, we expressed the problem of analysis and researchers' choice. Secondly, we identified the inclusion and exclusion criteria. Thirdly, we consulted the academic databases, filtered the studies according to our selection criteria, and chose suitable papers. Fourthly, we discussed and analysed the findings, and finally, we reported the results. 


\section{Definition of keywords}

In the titles, abstracts, and keywords fields, we looked for the term "Physical Internet". Given the subject's specificity, this was the only word appropriate for a complete quest. Next, we set the period of the search from 2011 to the beginning of 2021. We chose 2011 as the starting year because the PI was systematically discussed and introduced in this year.

\section{Inclusion and Exclusion Criteria}

The first aim was to screen publications based on their titles, abstracts, and keywords. During this process, we only included publications with an exclusive focus on the PI and excluded those concerned with the Digital Internet's physical infrastructure or device hardware. We also reviewed the chosen publications' sources to help to identify further publications. Since several databases were used, we merged all publications and eliminated the duplicates. The publications in question were read very carefully and debated between the reviewers until an agreement was reached.

\section{Data search}

We identified three sub-steps in the data search process. The first concerns the selection of databases. We chose Google Scholar, Web of Science, Scopus, Springer, Science Direct, and Wiley Online Library as our root databases. We then started searching in the different databases using the keywords identified in this first phase, i.e., "Physical Internet" and combined it with "logistics," "transport," or "freight." In the first stage, we collected the first list of publications based on the title and abstract.

\section{Selection of the most relevant papers}

In this stage, we implemented a filter based on the inclusion/exclusion criterion to identify all potentially relevant publications. This task attempts to rank the most important publications that dealt with the PI concept and exclude irrelevant studies. We read each paper during the selection phase and checked the list of references for each item to identify potentially relevant publications. After the full-content reading, 59 publications were identified as relevant for the final review and analysis.

\section{Coding process of selected papers}

We developed a coding framework based on the study objectives raised in the introduction following three main axes:

\section{Axis 1: Themes}

After the full-content reading and analysis of all selected publications, thirteen themes emerged:
- Detailed literature review of the PI

- Standardised modular containers, space/volume utilisation, handling cost, intelligent boxes.

- Inventory, optimised inventory levels, warehousing services, reduced inventory costs, maximised utilisation, etc.

- Distribution and transport, network optimisation, optimised routing, loading patterns, and truck scheduling

- Production, intelligent and dynamic manufacturing

- Dynamic pricing in the PI

- Auction trading in the PI

- Interconnected city logistics

- Cloud logistics platform

- Synchromodality

- Blockchain technology for the PI

- Horizontal collaboration in the PI

Axis 2: Decision-making level

- $\quad$ Strategic level: L1

- Tactical level: L2

- Operational level: L3

Table 1. Decision-making level

\begin{tabular}{|l|l|c|}
\hline \multirow{3}{*}{ Levels } & L1 & strategic level \\
\cline { 2 - 3 } & L2 & tactical level \\
\cline { 2 - 3 } & L3 & operational level \\
\hline
\end{tabular}

Axis 3: Sustainability dimensions

- Economic performance (D1)

- Environmental performance (D2)

- Social performance (D3)

Table 2. Sustainability dimensions

\begin{tabular}{|c|c|c|}
\hline \multirow{3}{*}{ Dimensions } & D1 & Economic \\
\cline { 2 - 3 } & D2 & Social \\
\cline { 2 - 3 } & D3 & Environmental \\
\hline
\end{tabular}

\section{RESULTS AND FINDINGS}

\section{Publications per year}

Fig. 1. depicts the annual distribution of the selected publications. In recent years, $76 \%$ of the studies were written since 2016, and the number of publications experienced inconsistent growth. We identified twelve articles (20\%) published in 2020 and the beginning of 2021; thus, we can expect further progress in the research areas for this year and the next coming years.

\section{Publications by journal}

The selected publications were published in 39 international journals. As shown in Fig. 2. the International Journal of Production Research published over $38 \%$ of the publications, followed by 
Table 3. Classification of the literature based on the PI themes, the decision levels, and the sustainability dimensions

\begin{tabular}{|c|c|c|c|}
\hline Authors & Themes & Levels & Dimensions \\
\hline [9] & \multirow{6}{*}{ Conceptualization of PI } & L1 & $\mathrm{D} 1+\mathrm{D} 2+\mathrm{D} 3$ \\
\hline$[5]$ & & L1 & $\mathrm{D} 1+\mathrm{D} 2+\mathrm{D} 3$ \\
\hline [17] & & L1 & $\mathrm{D} 1+\mathrm{D} 2+\mathrm{D} 3$ \\
\hline [18] & & L1 & $\mathrm{D} 1+\mathrm{D} 2+\mathrm{D} 3$ \\
\hline [19] & & L1 & $\mathrm{D} 1+\mathrm{D} 2+\mathrm{D} 3$ \\
\hline [20] & & L1 & $\mathrm{D} 1+\mathrm{D} 2+\mathrm{D} 3$ \\
\hline [11] & \multirow{4}{*}{ Detailed literature review of PI } & & \\
\hline [10] & & & \\
\hline [12] & & & \\
\hline [13] & & & \\
\hline [21] & \multirow{7}{*}{$\begin{array}{l}\text { Standardised modular containers- } \\
\text { space/volume } \\
\text { utilisation, handling cost, intelligent containers. }\end{array}$} & $\mathrm{L} 1+\mathrm{L} 2+\mathrm{L} 3$ & $\mathrm{D} 1+\mathrm{D} 2+\mathrm{D} 3$ \\
\hline [22] & & L3 & $\mathrm{D} 1+\mathrm{D} 2+\mathrm{D} 3$ \\
\hline [23] & & L3 & $\mathrm{D} 1+\mathrm{D} 2+\mathrm{D} 3$ \\
\hline [24] & & L1 & $\mathrm{D} 1+\mathrm{D} 2+\mathrm{D} 3$ \\
\hline [8] & & L3 & $\mathrm{D} 1+\mathrm{D} 2+\mathrm{D} 3$ \\
\hline [25] & & L3 & $\mathrm{D} 1+\mathrm{D} 3$ \\
\hline [26] & & $\mathrm{L} 2$ & D1+D3 \\
\hline [27] & \multirow{7}{*}{$\begin{array}{c}\text { Inventory_optimised inventory levels, } \\
\text { warehousing } \\
\text { services, reduced inventory costs, maximised } \\
\text { utilisation, etc. }\end{array}$} & L2 & D1 \\
\hline [28] & & $\mathrm{L} 2+\mathrm{L} 3$ & D1 \\
\hline [29] & & L2 & D1 \\
\hline [30] & & $\mathrm{L} 2+\mathrm{L} 3$ & D1 \\
\hline [31] & & L2 & D1 \\
\hline [32] & & L2 & D1 \\
\hline [6] & & L3 & $\mathrm{D} 1+\mathrm{D} 2+\mathrm{D} 3$ \\
\hline [43] & \multirow{3}{*}{$\begin{array}{c}\text { Production - intelligent and dynamic } \\
\text { manufacturing }\end{array}$} & L2 & D1+D3 \\
\hline [44] & & L2 & D1 \\
\hline [45] & & L1 & $\mathrm{D} 1+\mathrm{D} 2+\mathrm{D} 3$ \\
\hline [46] & \multirow{2}{*}{ Dynamic pricing in PI } & L3 & $\mathrm{D} 1$ \\
\hline [47] & & L3 & D1 \\
\hline [48] & \multirow{3}{*}{ Auction trading in PI } & $\mathrm{L} 2+\mathrm{L} 3$ & D1 \\
\hline [49] & & $\mathrm{L} 2$ & $\mathrm{D} 1+\mathrm{D} 3$ \\
\hline [50] & & L2 & D1 \\
\hline [33] & \multirow{17}{*}{$\begin{array}{l}\text { Distribution and transport-network } \\
\text { optimisation, } \\
\text { optimised routing, loading patterns, truck } \\
\text { scheduling, etc. }\end{array}$} & L2+L3 & D1+D3 \\
\hline [34] & & L3 & D1 \\
\hline [35] & & $\mathrm{L} 2+\mathrm{L} 3$ & $\mathrm{D} 1+\mathrm{D} 2+\mathrm{D} 3$ \\
\hline [36] & & L3 & $\mathrm{D} 1+\mathrm{D} 2+\mathrm{D} 3$ \\
\hline [37] & & $\mathrm{L} 2+\mathrm{L} 3$ & D1 \\
\hline [38] & & $\mathrm{L} 2+\mathrm{L} 3$ & D1 \\
\hline [39] & & L3 & D1 \\
\hline [15] & & L1 & $\mathrm{D} 1+\mathrm{D} 2+\mathrm{D} 3$ \\
\hline [40] & & L1 & $\mathrm{D} 1+\mathrm{D} 2+\mathrm{D} 3$ \\
\hline [41] & & L3 & D1 \\
\hline [42] & & $\mathrm{L} 1+\mathrm{L} 2+\mathrm{L} 3$ & D2 \\
\hline [51] & & $\mathrm{L} 1+\mathrm{L} 2+\mathrm{L} 3$ & $\mathrm{D} 1+\mathrm{D} 2+\mathrm{D} 3$ \\
\hline [52] & & L3 & $\mathrm{D} 1+\mathrm{D} 2+\mathrm{D} 3$ \\
\hline [53] & & L3 & $\mathrm{D} 1+\mathrm{D} 2+\mathrm{D} 3$ \\
\hline [54] & & L3 & $\mathrm{D} 1+\mathrm{D} 2+\mathrm{D} 3$ \\
\hline [55] & & L1 & $\mathrm{D} 1+\mathrm{D} 2+\mathrm{D} 3$ \\
\hline$[56]$ & & L1 & $\mathrm{D} 1+\mathrm{D} 2+\mathrm{D} 3$ \\
\hline [57] & \multirow{2}{*}{ Cloud logistics platform } & L1 & $\mathrm{D} 1+\mathrm{D} 2+\mathrm{D} 3$ \\
\hline [58] & & L3 & $\mathrm{D} 1+\mathrm{D} 3$ \\
\hline [59] & \multirow{2}{*}{ Synchromodality } & L3 & D1+D3 \\
\hline [60] & & L3 & D1 \\
\hline [61] & \multirow{4}{*}{ Blockchain technology for PI } & $\mathrm{L} 2$ & $\mathrm{D} 1+\mathrm{D} 2+\mathrm{D} 3$ \\
\hline [62] & & L1 & $\mathrm{D} 1+\mathrm{D} 2+\mathrm{D} 3$ \\
\hline [7] & & L1 & $\mathrm{D} 1+\mathrm{D} 2+\mathrm{D} 3$ \\
\hline [63] & & L1 & $\mathrm{D} 1+\mathrm{D} 2+\mathrm{D} 3$ \\
\hline [64] & \multirow{2}{*}{ Horizontal collaboration in PI } & L2 & $\mathrm{D} 1+\mathrm{D} 2+\mathrm{D} 3$ \\
\hline [4] & & L1 & $\mathrm{D} 1+\mathrm{D} 2+\mathrm{D} 3$ \\
\hline
\end{tabular}


the Journal of Business and Logistics, and the Journal of Intelligent Manufacturing. The rest of publications were published in 36 separate journals. These journals include, among others, "Transportation Journal," "Journal of Physical Distribution and Logistics Management," "The International Journal of Logistics Management," "International Journal of Advanced Logistics," "International Journal of Signal Processing, Image Processing, and Pattern Recognition," "Journal of Enterprise Information Management," "International Journal of Logistics Systems and Management," "International Journal of Computer Integrated Manufacturing" and "International Journal of Transport Development and Integration. “

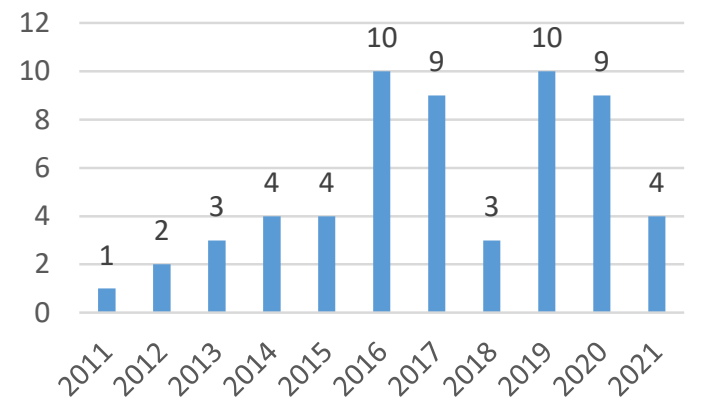

Figure 1. The annual distribution of selected publications

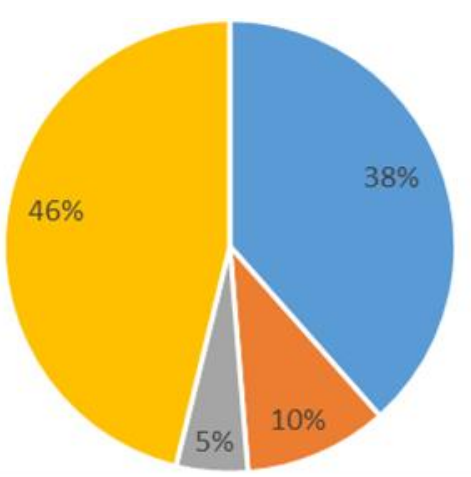

- International Journal of Production Research

- Journal of Business Logistics

- Journal of Intelligent Manufacturing

= Others

Figure 2. The distribution of publications according to journals

\section{Analysis of coding framework}

\section{A. Themes of PI}

Several previous reviews focused on diverse PI themes, such as [4], [12]. In our study, we considered these themes. Additionally, we considered other categories in our coding framework, namely decision levels and sustainability dimensions, aiming to inform researchers on how the decisions taken within the PI implementation and the impact of this paradigm on logistics sustainability.

\section{B. Decision levels}

Freight transport networks are dynamic systems containing various human, material, and capital resources that increased complexities of decisions and management strategies. According to [65], various freight networks need to be structured on three levels:

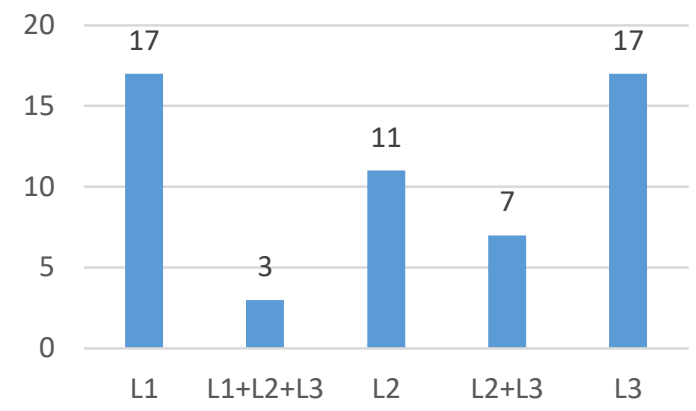

Figure 3. Distribution of studies according to the decision-making levels addressed

\section{Strategic level}

The strategic level relates to the business strategy that typically involves top management and needs considerable long-term commitment. At this decision level, the fundamental choices apply to the facilities required to ensure the movement of goods in diverse modes to reduce transportation costs [66]. Several difficulties arise at the strategic level, such as selection of the terminal site (e.g., multimodal cross-docking station), platform facilities, the construction of additional lines (roads, railways), and the demolition of specific infrastructure. On the one hand, we found out that many authors conceptualise the PI by using a business model innovation strategy, which led us to classify their publications into the strategic level of decisionmaking. For example, [9] introduces an audacious paradigm on how the physical object of transport moves forward and encourages the replication of the Digital Internet ambition to achieve sustainable global logistics. Later, [5] present a systematic description of the PI as an open global logistics infrastructure founded by encapsulation, interfaces, and protocols on physical, digital, and operational interconnection. [17] explore the potential impacts of PI-enabled business models on creativity. [18] discuss analogies and similarities between computer networks and logistic networks to examine the global structure of the interconnection of logistics networks that enables the PI. [20] offer an external and technical viewpoint on PI research to better inform researchers and policymakers. More recently, [19] suggest a model-based conceptual framework to introduce a feasible PI. 
On the other hand, various articles classified into the categories production, transportation, distribution, city logistics, cloud logistics, and blockchain technology discuss long-term decision planning during the PI implementation. For example, [15] and [40] cover the cross-docking problem by conceptualising a road-rail Pi-hub and a road-rail Pihub, respectively, showing how these models could contribute to economic, environmental, and social sustainability. [56] discuss the idea of Hyperconnected City Logistics, along with nine basic concepts, which provide a rich context for the design of effective urban logistics and transport systems. [57] aim to create a PI-enabled Building Information Modelling System (PI-BIMS) incorporating Auto-ID technology, BIM technology, and cloud storage. [62] introduce an intellectual paradigm using blockchain to provide a workable solution for the fundamental obstacles hindering the exchange of values and physical properties in logistics networks and decentralised management frameworks in the PI. Furthermore, [63] investigates the integration of the PI and Blockchain technologies in a conceptual paper and proposes several research directions for investigating the supply chain's institutional issues during the implementation of these two paradigms. [7] also examine blockchain as an enabling technology for the PI. The authors summarise the core requirements of the PI and explain how blockchain can be used to facilitate the implementation of the PI. [55] and [56] focus on conceptualising Interconnected city logistics, while [24] offers strategic analysis of freight and PIcontainers' total volumes and balances in a terminalto-terminal network. [45] explores the relations between the PI and Lean concepts using the Value Stream Map (VSM) method for their integrated use. At a broader level, [55] outline the possibilities for the PI's logistics performance from the EU perspective. This article details the systematic EU planning for the PI definition and the initial projects, ventures, tools that underpin the PI. [4] examine the reasons for accessing a PI network and the effectiveness of continuous cooperation variables in a PI network.

\section{Tactical level}

The tactical level is mainly concerned with a medium-term strategy. Tactical planning extends from a week to few months. At this stage, vehicle fleet management in multiple modes ensures the movement of goods from their sources to their destination. The tactical level is the degree to which we calculate the transport movements over time between authorities and terminals, inter-terminals, and between terminals and destinations. Tactical planning creates a travel strategy to ensure customer service satisfaction and resource efficiency. Therefore, the tactical decisions are primarily concerned with the collection of services to be provided, their frequency (or schedule), the allocation of demands for specific services in transportation, and terminal level policies (consolidation).

Several articles discuss the inventory problem at the tactical level. For instance, [31] study inventory efficiency over the PI network. Later, [32] aim to identify the latest research topics surrounding inventory management in a PI Network and how the PI can impact conventional stock control policies. [28] and [29] introduce PI disruption mitigation strategies. They carry out their study using a simulation-based optimisation model. [27] suggest a mixed integrated linear programming (MILP) formulation to assess the benefits of the PI from the cost efficiency perspective and to resolve the issue that combines the integrated output inventory allocation judgment with PI. [43] and [44] discuss the production manufacturing scheduling problem at medium-term decision making. In the former, the authors analyse a PI-enabled manufacturing executive system (PIMES) for a machine workshop. For the processing and synchronisation of real-time data, PIMES depends heavily on RFID and wireless communication networks. In the latter, the authors implement a big data analysis framework for PIbased logistics data generated by installing RFID readers, tags, and wireless communications networks on manufacturing stores.

Other articles focus on the auction trading in the PI, such as [49], [50]. [49] provide combinatorial frameworks for the auction of transport services in the PI sense. [50] aim to take advantage of the PI's open and uncertain environments to distribute and even reconnect containers for each centre. According to the authors, the PI can improve container routing and the use of logistics network tools. However, to achieve these objectives, [64] emphasise the need for horizontal coordination in the PI and for more effective solutions to any potential technological difficulties. In the context of horizontal partnerships and the PI, the authors also explore the perspectives, perceptions, values, and motives of transit operators in Austria to contribute to the Austrian physical network's early moves positively. In addition, [61] make a simulation model showing that blockchain technology can support IoT automated data processing and facilitate the implementation of the PI vision by introducing the very first hyper-connected logistics application.

\section{Operational level}

The operational level of decisions concerns shortterm management. This level includes diverse tasks such as assigning goods to liners and vehicles, container shipping, and truck routings.

Various researchers focus on container shipping issues. For example, [23] suggest a standard modular 
containers' production to minimise the number of boxes required for shipping goods using a decomposition-based solution methodology. [22] use a methodological engineering design process and explain how to create a modular and multifunctional loading unit by introducing a first real PI scenario in the fast-moving consumer goods industry and modelling the critical performance indicators (KPIs). Similarly, [8] propose a modular design consisting of three stages of characterisation, including the transport, storage, and packaging of PI containers. This paper outlines the proposed substance encapsulation transition and highlights significant opportunities and challenges for further research in industry and academia. [25] establish a new methodology for applying heuristic methods to address the liner shipping network design problem (LSNDP) using a mathematical model. Later, [26] concentrate on product development in the context of a global open logistics system, the PI, utilising a hyperconnected-mobile production mode. The study deals with the complex distribution of output units, allocation and sharing of resources.

Routing optimisation and transportation problems are the focus of several articles. For example, [39] highlight the efficient management of the PI for mobile resources, such as containers. In reality, management and storage of resources (containers) can cause imbalances in the logistic network, leading to starvation or unnecessary storage of logistic network nodes. For this reason, [36] present a $\pi$ container tracking device to create and hold the composite $\pi$-container practically $3 \mathrm{D}$ layout dynamically. [34] suggest a new method for routing based on the Internet BGP equivalent of BGP (the PI Border Gateway Protocol). To fix the issue of routing the PI-containers in the PI, the authors create this new protocol. Afterwards, [46], [47] present and explore a dynamic pricing model based on an auction mechanism for optimising transport service providers' bid prices in PI hubs. [41] define the best suitable hub for forwarding transport based on realtime traffic data. This article addresses the critical research questions of when and how to make routing decisions for road vehicles if the routing schemes within the PI take consider the data related to transport vacancies in real-time. [6] focus on integrated production-inventory-distribution planning in the PI for sustainability purposes. The key objective of their study is to thoroughly evaluate the efficiency of the PI-enabled integrated production-inventory-distribution model in the three sustainability dimensions.

Moreover, other studies discuss the interconnected city logistics (ICL) topic using simulation and mathematical models to solve some routing problems. To illustrate, [53] discuss PI-enabled urban transport and attempt to model essential organisational elements of the ICL initiative. [54] aim to explore an alternative approach to reverse ecommerce flows collection citywide, and [52] examine the potential for open mobility webs in an urban environment with e-commerce warehouses based on the PI concept.

Related to the theme Cloud logistics platform, [58] discuss crucial problems and difficulties with cloud logistics orders's deployment in China. We found two more articles that solve synchromodality issues with optimisation models. [59] examine how synchromodal transport can promote the modal transition to slower and more environmentally sustainable transport modes without sacrificing costs or responsiveness. [60] aim to extract rules for effective container allocation for inland services in real-time by analysing the solution structure of a centralised, offline system of optimisation used for historical results.

\section{Tactical and operational level}

Some other papers combine the tactical and organisational levels. For example, [30] solve a real problem in planning and distribution for multiple facilities that simultaneously maximise the effectiveness of decision-making in supply, inventory, demand, and distribution. [37] emphasise the difference between traditional logistics networks and the PI and characterised the PI by using a relatively more superficial $\mathrm{P} 2 \mathrm{P}$ optimal dispatch MILP. [38] aim to increase the railroad PI-hub's performance by minimising the distance to the dock by each container and the number of used trucks. The research concerns a PI allocation issue. [48] propose a scheduling solution for trolley loading and sale in PI-Auction logistics centre (ALC) systematically. [28], [29] suggest a revolutionary vendor-managed inventory strategy that uses the PI's flexible, universal, interconnected logistics structure. [35] aim to compare PI's efficiency with the traditional logistics method to measure the truck and driver routing advantages and drawbacks of PI with an explicit limit to the driver's maximum return time. [33] propose an efficient and reactive multi-agent system-based model (MAS) for the resolution of trucks and PI-containers grouping.

\section{All decisions level}

Other articles englobe all levels of decisions, such as [21], who highlight the importance of semantics in distribution and logistical at multiple stages of judgment to achieve various optimisations such as bundling and durability, enforcement, and travel optimisation, thereby increasing sustainability through optimal use of capacities. Additionally, [51] attempt to establish the latest concept of an interconnected multilayer decision-making system and a PI methodology. [42] outline a theoretical framework that describes various levels of acceptance and trust as a key component of "Human- 
computer interaction" in technology innovation and point out a risk of an artificial divide both at the level of individuals and of companies.

\section{Multiple freight transportation problems}

The PI is a new concept made to serve supply chain sustainability throughout the world. [5] argue that three forms of efficiencies impact logistics and transport systems: social, economic, and environmental. First, the PI's societal goal is to improve the high quality of life of logistics personnel and the global population by improving physical items' timely accessibility and mobility. Second, the economic ambition is to sustainably reduce largescale purchase strategies, international monetary burden, while freeing up enormous gains in companies' production. Third, the environmental intention is to reduce global greenhouse gas emissions caused by logistics, energy consumption, pollution, traffic, and waste materials.

The present state of the PI research focuses on studying the combination of economic and environmental goals. However, in the extant literature, the social element is less explored (Fig. 4.).

Fig. 4. shows that the PI research mainly centres on the economic dimension of sustainability. Of the 59 papers listed in Table 1, 17 papers highlight the financial targets of the PI. Seven papers analyse the PI's environmental and economic aspects, and 30 articles deal with the PI concept in the context of the three dimensions of sustainability. Finally, there is only one contribution to the PI concept from the perspective of social sustainability, and no papers deal with the PI and the convergence between the social and environmental and economic dimensions.

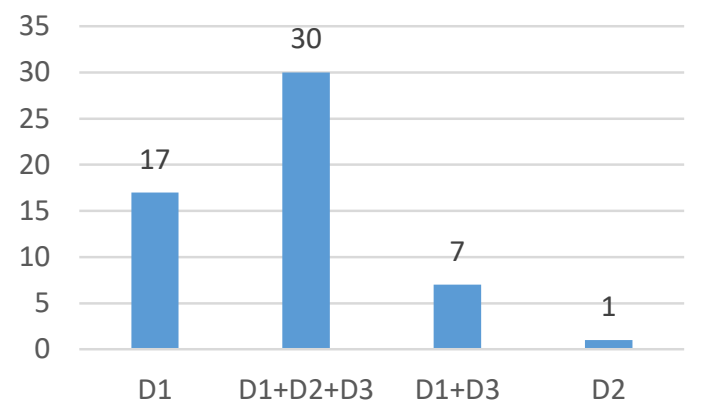

Figure 4. Count of dimensions

\section{The economic dimension}

The most crucial economic issue is that the logistics and freight traffic prices rise at the global level. To solve this dilemma, numerous articles study the economics dispute on a tactical level. For example, [31] use objective-oriented modelling experiments to minimise the overall transportation and inventory costs. [50] utilise a simulation model for assigning and allocating containers at the hub to carriers while optimising container routing and using logistics network tools. [32] analyse an organisation's effect on inventory levels and costs using a multi-method simulation modelling software developed by XJ technologies (www.anylogic.com). [28], [29] examine how stock models using the PI react to intervention and durability in supply chain systems. The topic is explored through an advanced numerical analysis using the simulation-based optimisation model. [27] present a mathematical model to resolve the issue that incorporates an optimised production-inventory-distribution decision to measure the benefits of PI from the cost efficiency viewpoint. Likewise, plenty of researchers are interested in disbanding the operational issues. [39] conduct active research to optimise the freight by exposing the efficiency handling resources issues. [60] aim to develop guidelines for the efficient distribution of containers to inland installations in real-time by evaluating a critical offline optimisation approach used for historical outcomes. [34] focus on truck-transported PI-containers and suggest a new routing method to optimise road transportation. [46], [47] present and address a dynamic price model targeted at a service provider that offers a mechanism to optimise PI hubs' cost. The results and observations provide carriers with instructive guidelines on real-life pricing. [41] interview experts and make a qualitative analysis on artefact routing. More than that, some studies evaluate the economic issue at both the tactical and operational levels simultaneously. [30] use a mathematical model to minimise the total cost of production, inventory, and distribution. [37] suggest a cost-minimising mathematical model to minimise handling and delivery cost. [38] use a multi-agent system model (optimisation models) to reduce container travel distance to the dock. [48] conduct simulation experiments with a timely operation scheduling model to reduce logistics and labour costs. [28], [29] propose a retailer's creative inventory policy to minimise inventory levels, overall expense, and distances.

\section{The environmental dimension}

The harmful impacts of freight transport on the environment (greenhouse gases, energy consumption, noise) are growing amid the fact that nations and governments are seeking to enhance environmental quality, commit substantial budgets to environmental safety, and reduce the emission rate of air pollutants.

Several papers address economic and environmental issues, such as [49], who attempt to reduce the cost of transport services on the PI and greenhouse gas emissions by using a mathematical model. [43] suggest PIMES, an information system that can reduce paperwork and enhance information 
flow with an analytical data model. [43] propose a smart box compatible with PSS to provide revolutionary eco-logistics and safe packaging operation and minimise the logistics costs and emissions. [25] highlight the global CO2 emissions problem due to the large volumes transported and propose a heuristic for solving the liner shipping network design problem to maximise an organisation's profit. [59] research how synchro modal transport support a slower but eco-friendlier modal transition without compromising costs or responsiveness. [33] suggest models that discuss two of the three pillars: sustainable growth and the economic and environmental costs. The proposed models minimise the average expense from an economic point of view using a minimal number of cars and delay vehicles, leading to financial fines from the transport providers. From an environmental point of view, the two models decrease the number of used wagons, thus reducing $\mathrm{CO} 2$ emissions by the rail locomotive and the distance travelled for PI containers. [26] suggest a two-target optimisation model that minimises the cost and greenhouse gas of a producer to support its clients through the availability of open fabs.

\section{The social dimension}

The transportation of goods often causes social problems, such as road safety, lack of quick, efficient, accessible goods mobility. Furthermore, there is a shortage of stability, protection, and security in working conditions in the freight transport sector. We found that few studies address social issues facing logistics. For example, [42] discuss measures to improve human-computer interaction, especially the capacity of individuals and companies to implement state-of-the-art techniques and prevent an artificial divide, thus increasing social sustainability at an operational, tactical and strategic level. In addition, these articles attribute social problems along with financial and environmental issues [5], [9], [17], [18]. Further, [10], [19] evaluate and optimise certain aspects of the early conceptualisation of PI. [8], [21 - 24] develop the standardised modular containers to make the transport and handling of $\pi$-containers very easy. [6], [14], [15], [35], [36] seek to refine delivery network routing by reducing container journey distance that would positively affect carriers, lower congestion, and improve living conditions. [45] aims to reduce the number of trucks on roads and improve customers' quality of life and the logistics sector workers. [51 - 56] discuss the city logistics sector that offers less infrastructure usage and fewer jams, making consumers and community happier and distribution quicker. [7], [61 - 63] analyse how blockchain technology can improve visibility, security, and traceability of products through its decentralised and trusting mechanisms.
Researchers and professionals were prompted to carry out studies that aim to reduce various adverse effects of the lack of sustainability and unreliable traditional transport networks. Several perceptions have therefore arisen in recent years. Three creative theoretical frameworks are most effective in achieving sustainability, including collaborative logistics, city-logistics (CL), and the PI.

\section{FUTURE RESEARCH}

Our analysis recommends several topics for future studies. Our literature review urges PI researchers to focus more on theory and highlights the importance of a clear PI strategy in logistics. Addressing 'PI themes,' the 'decision stages', and the 'sustainability aspects' mentioned here will possibly provide a sound base for assessing the actual PI adoption and its impact on logistics sustainability. We took a closer look at critical and diverse topics regarding decision-making levels and sustainability aspects to provide recommendations for future research.

The PI literature addresses numerous strategy decisions ranging from strategic to operational. This demonstrates how the PI is relevant and beneficial to the logistics and transport sector. Researchers may expand future studies by evaluating the relationship between the logistical service providers and external factors, such as clients and competitors. In reality, consumer involvement through a collective strategy will improve business efficiency in general.

Environmental and social considerations have been relevant to broaden the economic implications and include other sustainable growth dimensions. This highlights the significance and value of incorporating facets of sustainable growth into planning decisions for logistics performance. However, social sustainability has been poorly considered compared to economic and environmental sustainability. This finding indicates that social dimensions must be studied to resolve all issues of sustainability holistically. Due to its qualitative nature, social sustainability is concerned with the employer's health and well-being issues. Therefore, future studies should also focus on quantifying this sustainability dimension. Integrating corporate social responsibility (CSR) in planning and policy-making helps researchers to properly determine the PI's effect on customers, workers, and the local population. It also allows identifying the detrimental influence of the PI on human lives to as well.

Usually, environmental sustainability is limited to measuring transportation $\mathrm{CO} 2$ emissions. The ecological evaluation revolves around assessing direct pollution from installations and inventory, waste volume and energy use.

Since the PI is still a fresh and novel concept, the lack of data is a barrier to further scientific 
advancement in this field. More studies are nevertheless necessary taking into account real-life cases. The collaboration between researchers and managers may result in insightful findings. Academics and researchers may study various reallife cases in the future. For example, this involves the validation of unimodal (road-based) and multimodal (road-to-rail) PI hubs, the identification of the next best appropriate hubs for onward transport based on real-time traffic data, the validation of the proposed design and types of PI containers, the confirmation of the proposed auction mechanisms, the optimisation of capacity utilisation for PI carriers in PI hubs, and validation of the proposed legal frameworks for horizontal collaboration. Most of the studies are concentrating on unimodal (road-based) and multimodal (road-to-rail) PI hubs. As a result, there is a lack of studies addressing maritime and air transportation and conceptualising multimodal road and rail to sea hubs. This will help to effectively connect multimodal road to air PI hubs.

[19] recommend a paradigm based exclusively on transportation issues, not those like network inventory management. Thus, future research may incorporate the PI's decisions, transport, and inventory. In addition, PI models may consider additional features of the PI. For example, cost and time may depend on other parameters such as shipping size, node driving times, capacity, speed limits, carrier limits. [28], [29] also affirm that more research is necessary to compare performance with optimal management decisions and procurement methods for distribution systems.

There is a need for future research to examine a network closer to a real-life case of connectable supply networks with more suppliers and employees. Some studies on how the inventory control models, such as operational models and decision-making tools, the analysis of management issues, and the adaptability of models to different systems are required. The development of awareness of business and organisational models used by the PI will significantly improve the network's vision and cooperation.

When several organisations, such as businesses, governments, and customers, are likely to use a common infrastructure, one of the future tasks will be to create specifications and architectures that will benefit each party in the logistics network. Therefore, we assume that criteria related to the conceptual architecture must be met to implement the PI, including the development of more operational processes, incentives, and reliable sharing of data.

The combination of blockchain and the PI will ensure trust, traceability, clarity, originality, and honesty. However, many studies are also required for the efficient integration of the technology in the
PI environment. Scalability is one of the biggest challenges of blockchain. Scalability is the ability of a system to cope and perform well while increasing or expanding the network performance. The design of an overall logistics network necessitates high performance consistent with the large implementation of the PI. Perhaps the blockchain network's scalability and performance are unable to handle a sizeable interconnected PI network in its current situation.

Future-oriented logistical problems are highly prevalent. They include the excessive use of storage and transportation facilities, their costs and corresponding $\mathrm{CO}_{2}$ reductions, the complexities of logistics activities, and increased process inefficiencies. Technologies like Big Data, the Internet of Things, 3D Printing, or other Industry 4.0 innovations have become part of logistics. Besides, to be competitive in the future market, organisations must deal with these developments.

\section{CONCLUSION}

The PI has been a highly promising and evolving freight transport paradigm over the last few years. This paper systematically reviews the PI literature, which is published over the last decade. This review has shown that the PI is a global logistics and holistic concept that solves many problems associated with the current supply chain and logistics models. The growth in the PI literature and the capacity of this paradigm for disrupting supply chain and logistics techniques are examined, and several recommendations for future research are highlighted.

In this study, the latest state-of-the-art is reviewed and assessed. We categorise the literature according to three major classes: PI themes, decision levels, and sustainability dimensions. Next, we identify a range of research propositions based on the study's findings, which are worth exploring in future work to ensure logistics sustainability. The study findings show that the main decisions taken in the implementation of the PI are the tactical and operational decisions, with over $65 \%$ of selected publications addressing these two decision levels. Moreover, there is little emphasis on cooperation between external parties such as logistics service providers, competitors and customers. In addition, the emphasis is placed more on economic and environmental sustainability than on social sustainability.

This study proposes a novel coding framework to systematise the literature and outlines several future research directions for logistics and supply chain management scholars. Further PI research will likely lead to motivate the implementations of the PI and more development of technologies that will improve efficiency, performance and sustainable 
development of supply chains and logistics, thereby benefitting the standard of living of the society.

\section{AUTHOR CONTRIBUTIONS}

S. Ben Neila: Conceptualization, Experiments, Theoretical analysis.

A. Rejeb: Finite element modelling, Writing, Review and editing.

P. Németh: Supervision, Review and editing.

\section{REFERENCES}

[1] A. Scipioni, M. Mastrobuono, A. Mazzi, and A. Manzardo, 'Voluntary GHG management using a life cycle approach. A case study', J. Clean. Prod., vol. 18, no. 4, pp. 299-306, Mar. 2010.

https://doi.org/10.1016/j.jclepro.2009.10.007

[2] J. Samuelson, The Six New Rules of Business: Creating Real Value in a Changing World. Berrett-Koehler Publishers, 2021.

[3] M. Henke and H. Kohl, Sustainability in Global Value Chains: Measures, Ethics and Best Practices for Responsible Businesses. Kogan Page Publishers, 2021.

[4] M. Plasch, S. Pfoser, M. Gerschberger, R. Gattringer, and O. Schauer, 'Why Collaborate in a Physical Internet Network?-Motives and Success Factors', J. Bus. Logist., vol. 42, no. 1, pp. 120-143, Mar. 2021. https://doi.org/10.1111/jbl.12260

[5] B. Montreuil, R. D. Meller, and E. Ballot, 'Physical Internet Foundations', IFAC Proc. Vol., vol. 45, no. 6, pp. 26-30, May 2012. https://doi.org/10.3182/20120523-3-RO2023.00444

[6] X. Peng, S. Ji, and T. Ji, 'Promoting sustainability of the integrated productioninventory-distribution system through the Physical Internet', Int. J. Prod. Res., vol. 58, no. 22, pp. 6985-7004, Nov. 2020. https://doi.org/10.1080/00207543.2019.16879 $\underline{53}$

[7] H. R. Hasan, K. Salah, R. Jayaraman, I. Yaqoob, and M. Omar, 'Blockchain Architectures for Physical Internet: A Vision, Features, Requirements, and Applications', IEEE Netw., vol. 35, no. 2, pp. 174-181, Mar. 2021.

https://doi.org/10.1109/MNET.021.2000442

[8] B. Montreuil, E. Ballot, and W. Tremblay, 'Modular Design of Physical Internet Transport, Handling and Packaging Containers', p. 30.

[9] B. Montreuil, 'Toward a Physical Internet: meeting the global logistics sustainability

\section{DisClOSURE STATEMENT}

The authors declare that they have no known competing financial interests or personal relationships that could have appeared to influence the work reported in this paper.

\section{ORCID}
A. Rejeb http://orcid.org/000-0002-2817-5377
P. Németh http://orcid.org/0000-0002-7825-6773

grand challenge', Logist. Res., vol. 3, no. 2, pp. 71-87, May 2011. https://doi.org/10.1007/s12159-011-0045-X

[10] H. Treiblmaier, K. Mirkovski, and P. B. Lowry, 'Conceptualizing the Physical Internet: Literature Review, Implications and Directions for Future Research', Social Science Research Network, Rochester, NY, SSRN Scholarly Paper ID 2861409, May 2016. Accessed: Jul. 07, 2021. https://papers.ssrn.com/abstract=2861409

[11] T. Ambra, A. Caris, and C. Macharis, 'Towards freight transport system unification: reviewing and combining the advancements in the physical internet and synchromodal transport research', Int. J. Prod. Res., vol. 57, no. 6, pp. 1606-1623, Mar. 2019. https://doi.org/10.1080/00207543.2018.14943 $\underline{92}$

[12] H. Treiblmaier, K. Mirkovski, P. B. Lowry, and Z. G. Zacharia, 'The physical internet as a new supply chain paradigm: a systematic literature review and a comprehensive framework', Int. J. Logist. Manag., vol. 31, no. 2, pp. 239-287, May 2020. https://doi.org/10.1108/IJLM-11-2018-0284

[13] K. Jharni, M. Hlyal, and J. El Alami, 'Physical Internet: Literature review and research opportunities', IOP Conf. Ser. Mater. Sci. Eng., vol. 827, p. 012044, Jun. 2020.

https://doi.org/10.1088/1757899X/827/1/012044

[14] B. Montreuil, R. D. Meller, and E. Ballot, 'Physical Internet Foundations', in Service Orientation in Holonic and Multi Agent Manufacturing and Robotics, T. Borangiu, A. Thomas, and D. Trentesaux, Eds. Berlin, Heidelberg: Springer, 2013, pp. 151-166. https://doi.org/10.1007/978-3-642-35852-4_10

[15] E. Ballot, B. Montreuil, and C. Thivierge, 'Functional Design of Physical Internet Facilities: A Road-Rail Hub', p. 36.

[16] D. Tranfield, D. Denyer, and P. Smart, 'Towards a Methodology for Developing Evidence-Informed Management Knowledge 
by Means of Systematic Review', Br. J. Manag., vol. 14, no. 3, pp. 207-222, Sep. 2003. https://doi.org/10.1111/1467-8551.00375

[17] B. Montreuil, J.-F. Rougès, Y. Cimon, and D. Poulin, 'The Physical Internet and Business Model Innovation', Technol. Innov. Manag. Rev., p. 6, 2012.

[18] R. Sarraj, E. Ballot, S. Pan, and B. Montreuil, 'Analogies between Internet network and logistics service networks: challenges involved in the interconnection', J. Intell. Manuf., vol. 25, no. 6, pp. 1207-1219, Dec. 2014. https://doi.org/10.1007/s10845-012-0697-7

[19] C. Dong and R. Franklin, 'From the Digital Internet to the Physical Internet: A Conceptual Framework With a Stylized Network Model', J. Bus. Logist., vol. 42, no. 1, pp. 108-119, Mar. 2021. https://doi.org/10.1111/jbl.12253

[20] H. Sternberg and A. Norrman, 'The Physical Internet - review, analysis and future research agenda', Int. J. Phys. Distrib. Logist. Manag., vol. 47, no. 8, pp. 736-762, Sep. 2017. https://doi.org/10.1108/IJPDLM-12-2016$\underline{0353}$

[21] W. Hofman, M. Punter, H. Bastiaansen, E. Cornelisse, and S. Dalmolen, 'Semantic technology for enabling logistics innovations towards Intelligent Cargo in the Physical Internet', Int. J. Adv. Logist., pp. 1-12, Apr. 2016.

https://doi.org/10.1080/2287108X.2016.11443 $\underline{58}$

[22] C. Landschützer, F. Ehrentraut, and D. Jodin, 'Containers for the Physical Internet: requirements and engineering design related to FMCG logistics', Logist. Res., vol. 8, no. 1, p. 8, Dec. 2015. https://doi.org/10.1007/s12159-015-0126-3

[23] Y.-H. Lin, R. D. Meller, K. P. Ellis, L. M. Thomas, and B. J. Lombardi, 'A decomposition-based approach for the selection of standardized modular containers', Int. J. Prod. Res., vol. 52, no. 15, pp. 46604672, Aug. 2014. https://doi.org/10.1080/00207543.2014.88346 8

[24] H. S. Sternberg and M. Denizel, 'Toward the Physical Internet-Logistics Service Modularity and Design Implications', J. Bus. Logist., vol. 42, no. 1, pp. 144-166, Mar. 2021. https://doi.org/10.1111/jbl.12261

[25] A. Krogsgaard, D. Pisinger, and J. Thorsen, 'A flow-first route-next heuristic for liner shipping network design', Networks, vol. 72, no. 3, pp. 358-381, Oct. 2018. https://doi.org/10.1002/net.21819

[26] C. Fergani, A. El Bouzekri El Idrissi, S. Marcotte, and A. Hajjaji, 'Optimization of hyperconnected mobile modular production toward environmental and economic sustainability', Environ. Sci. Pollut. Res., vol. 27, no. 31, pp. 39241-39252, Nov. 2020. https://doi.org/10.1007/s11356-020-09966-9

[27] S. Ji, X. Peng, and R. Luo, 'An integrated model for the production-inventorydistribution problem in the Physical Internet', Int. J. Prod. Res., vol. 57, no. 4, pp. 1000-1017, Feb. 2019. https://doi.org/10.1080/00207543.2018.14978 $\underline{18}$

[28] Y. Yang, S. Pan, and E. Ballot, 'Innovative vendor-managed inventory strategy exploiting interconnected logistics services in the Physical Internet', Int. J. Prod. Res., vol. 55, no. 9, pp. 2685-2702, May 2017. https://doi.org/10.1080/00207543.2016.12758 71.

[29] Y. Yang, S. Pan, and E. Ballot, 'Mitigating supply chain disruptions through interconnected logistics services in the Physical Internet', Int. J. Prod. Res., vol. 55, no. 14, pp. 3970-3983, Jul. 2017.

https://doi.org/10.1080/00207543.2016.12233 $\underline{79}$

[30] $\bar{M}$. Darvish, H. Larrain, and L. C. Coelho, 'A dynamic multi-plant lot-sizing and distribution problem', Int. J. Prod. Res., vol. 54, no. 22, pp. 6707-6717, Nov. 2016. https://doi.org/10.1080/00207543.2016.11546 23

[31] S. Pan, M. Nigrelli, E. Ballot, and R. Sarraj, 'PERFORMANCE ASSESSMENT OF DISTRIBUTED INVENTORY IN PHYSICAL INTERNET', p. 17, 2013.

[32] S. Pan, M. Nigrelli, E. Ballot, R. Sarraj, and Y. Yang, 'Perspectives of inventory control models in the Physical Internet: A simulation study', Comput. Ind. Eng., vol. 84, pp. 122132, Jun. 2015. https://doi.org/10.1016/j.cie.2014.11.027

[33] T. Chargui, A. Bekrar, M. Reghioui, and D. Trentesaux, 'Proposal of a multi-agent model for the sustainable truck scheduling and containers grouping problem in a Road-Rail physical internet hub', Int. J. Prod. Res., vol. 58, no. 18, pp. 5477-5501, Sep. 2020. https://doi.org/10.1080/00207543.2019.16608 $\underline{25}$

[34] S. Gontara, A. Boufaied, and O. Korbaa, 'Routing the Pi-Containers in the Physical Internet using the PI-BGP Protocol', in 2018 IEEE/ACS 15th International Conference on Computer Systems and Applications (AICCSA), Aqaba, Oct. 2018, pp. 1-8. https://doi.org/10.1109/AICCSA.2018.861288 $\underline{5}$

[35] M. Fazili, U. Venkatadri, P. Cyrus, and M. Tajbakhsh, 'Physical Internet, conventional and hybrid logistic systems: a routing 
optimisation-based comparison using the Eastern Canada road network case study', Int. J. Prod. Res., vol. 55, no. 9, pp. 2703-2730, May 2017.

https://doi.org/10.1080/00207543.2017.12850 $\underline{75}$

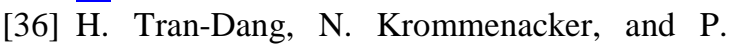
Charpentier, 'Containers monitoring through the Physical Internet: a spatial 3D model based on wireless sensor networks', Int. J. Prod. Res., vol. 55, no. 9, pp. 2650-2663, May 2017. https://doi.org/10.1080/00207543.2016.12062 $\underline{20}$

[37] U. Venkatadri, K. S. Krishna, and M. A. Ulku, 'On Physical Internet Logistics: Modeling the Impact of Consolidation on Transportation and Inventory Costs', IEEE Trans. Autom. Sci. Eng., vol. 13, no. 4, pp. 1517-1527, Oct. 2016. https://doi.org/10.1109/TASE.2016.2590823

[38] F. Walha, A. Bekrar, S. Chaabane, and T. M. Loukil, 'A railroad PI-hub allocation problem: Active and reactive approaches', Comput. Ind., vol. 81, pp. 138-151, Sep. 2016.

https://doi.org/10.1016/j.compind.2016.04.007

[39] J.-Y. Colin, M. Nakechbandi, and H. Mathieu, 'Management of mobile resources in Physical Internet logistic models', in 2015 4th International Conference on Advanced Logistics and Transport (ICALT), Valenciennes, France, May 2015, pp. 323-326. https://doi.org/10.1109/ICAdLT.2015.713659 $\underline{8}$

[40] B. Montreuil, R. D. Meller, C. Thivierge, and Z. Montreuil, 'Functional Design of Physical Internet Facilities: A Unimodal Road-Based Crossdocking Hub', p. 55.

[41] S. Kaup, A. Ludwig, and B. Franczyk, 'Design and Evaluation of Routing Artifacts as a Part of the Physical Internet Framework', p. 16.

[42] M. Klumpp and H. Zijm, 'Logistics Innovation and Social Sustainability: How to Prevent an Artificial Divide in Human-Computer Interaction', J. Bus. Logist., vol. 40, no. 3, pp. 265-278, Sep. 2019. https://doi.org/10.1111/jbl.12198

[43] R. Y. Zhong, H. Gong, C. Xu, and S. Lu, 'Physical Internet-Enabled Manufacturing Execution System for Intelligent Workshop Production', Int. J. Signal Process. Image Process. Pattern Recognit., vol. 9, no. 6, pp. 121-132, Jun. 2016. https://doi.org/10.14257/ijsip.2016.9.6.11

[44] R. Y. Zhong, C. Xu, C. Chen, and G. Q. Huang, 'Big Data Analytics for Physical Internet-based intelligent manufacturing shop floors', Int. J. Prod. Res., vol. 55, no. 9, pp. 2610-2621, May 2017.

https://doi.org/10.1080/00207543.2015.10860 $\underline{37}$
[45] V. Rodríguez Cornejo, Á. Cervera Paz, L. López Molina, and V. Pérez-Fernández, 'Lean Thinking to Foster the Transition from Traditional Logistics to the Physical Internet', Sustainability, vol. 12, no. 15, p. 6053, Jul. 2020. https://doi.org/10.3390/su12156053

[46] B. Qiao, S. Pan, and E. Ballot, 'Revenue optimization for less-than-truckload carriers in the Physical Internet: dynamic pricing and request selection', Comput. Ind. Eng., vol. 139, p. 105563, Jan. 2020. https://doi.org/10.1016/j.cie.2018.12.010

[47] B. Qiao, S. Pan, and E. Ballot, 'Dynamic pricing model for less-than-truckload carriers in the Physical Internet', J. Intell. Manuf., vol. 30, no. 7, pp. 2631-2643, Oct. 2019. https://doi.org/10.1007/s10845-016-1289-8

[48] X. T. R. Kong, J. Chen, H. Luo, and G. Q. Huang, 'Scheduling at an auction logistics centre with physical internet', Int. J. Prod. Res., vol. 54, no. 9, pp. 2670-2690, May 2016. https://doi.org/10.1080/00207543.2015.11171 $\underline{49}$

[49] I. B. Othmane, M. Rekik, and S. Mellouli, 'Impact of Shipper Collaboration on Carriers Selection in Reputation-Based Transportation Auctions', p. 9.

[50] S. Pan, X. Xu, and E. Ballot, 'Auction based transport services allocation in Physical Internet: a simulation framework', p. 13.

[51] D. Kubek and P. Więcek, 'An integrated multilayer decision-making framework in the Physical Internet concept for the City Logistics', Transp. Res. Procedia, vol. 39, pp. 221-230, 2019.

https://doi.org/10.1016/j.trpro.2019.06.02

[52] L. Zheng, 'Assessment of the physical internet enabled urban logistics using agent-based simulation', p. 26.

[53] I. Ben Mohamed, W. Klibi, O. Labarthe, J.-C. Deschamps, and M. Z. Babai, 'Modelling and solution approaches for the interconnected city logistics', Int. J. Prod. Res., vol. 55, no. 9, pp. 2664-2684, May 2017. https://doi.org/10.1080/00207543.2016.12674 12

[54] C. Chen, S. Pan, Z. Wang, and R. Y. Zhong, 'Using taxis to collect citywide E-commerce reverse flows: a crowdsourcing solution', Int. J. Prod. Res., vol. 55, no. 7, pp. 1833-1844, Apr. 2017.

https://doi.org/10.1080/00207543.2016.11732 $\underline{58}$

[55] Matusiewicz, 'Logistics of the FuturePhysical Internet and Its Practicality', Transp. J., vol. 59, no. 2, p. 200, 2020. https://doi.org/10.5325/transportationj.59.2.02 $\underline{00}$ 
[56] T. G. Crainic and B. Montreuil, 'Physical Internet Enabled Hyperconnected City Logistics', Transp. Res. Procedia, vol. 12, pp. 383-398, 2016. https://doi.org/10.1016/j.trpro.2016.02.074

[57] K. Chen, G. Xu, F. Xue, R. Y. Zhong, D. Liu, and W. Lu, 'A Physical Internet-enabled Building Information Modelling System for prefabricated construction', Int. J. Comput. Integr. Manuf., vol. 31, no. 4-5, pp. 349-361, Apr. 2018.

https://doi.org/10.1080/0951192X.2017.13790 $\underline{95}$

[58] Y. Zhang, S. Liu, Y. Liu, and R. Li, 'Smart boxenabled product-service system for cloud logistics', Int. J. Prod. Res., vol. 54, no. 22, pp. 6693-6706, Nov. 2016. https://doi.org/10.1080/00207543.2015.11348 $\underline{40}$

[59] N. Lemmens, J. Gijsbrechts, and R. Boute, 'Synchromodality in the Physical Internet dual sourcing and real-time switching between transport modes', Eur. Transp. Res. Rev., vol. 11, no. 1, p. 19, Dec. 2019. https://doi.org/10.1186/s12544-019-0357-5

[60] B. van Riessen, R. R. Negenborn, and R. Dekker, 'Real-time container transport planning with decision trees based on offline obtained optimal solutions', Decis. Support Syst., vol. 89, pp. 1-16, Sep. 2016. https://doi.org/10.1016/j.dss.2016.06.004

[61] Q. Betti, R. Khoury, S. Hallé, and B. Montreuil, 'Improving Hyperconnected Logistics with Blockchains and Smart Contracts',
ArXiv190403633 Cs, Apr. 2019, Accessed: Jul. 07, 2021. http://arxiv.org/abs/1904.03633

[62] T. Meyer, M. Kuhn, and E. Hartmann, 'Blockchain technology enabling the Physical Internet: A synergetic application framework', Comput. Ind. Eng., vol. 136, pp. 5-17, Oct. 2019. https://doi.org/10.1016/j.cie.2019.07.006

[63] H. Treiblmaier, 'Combining Blockchain Technology and the Physical Internet to Achieve Triple Bottom Line Sustainability: A Comprehensive Research Agenda for Modern Logistics and Supply Chain Management', Logistics, vol. 3, no. 1, p. 10, Feb. 2019. https://doi.org/10.3390/logistics3010010

[64] L. Simmer, S. Pfoser, M. Grabner, O. Schauer, and L. M. Putz, 'From horizontal collaboration to the physical Internet - A case study from austria', Int. J. Transp. Dev. Integr., vol. 1, no. 2, pp. 129-136, Jan. 2017. https://doi.org/10.2495/TDI-V1-N2-129-136

[65] T. G. Crainic and G. Laporte, 'Planning models for freight transportation', Eur. J. Oper. Res., vol. 97, no. 3, pp. 409-438, Mar. 1997. https://doi.org/10.1016/S03772217(96)00298-6

[66] T. G. Crainic and K. H. Kim, 'Chapter 8 Intermodal Transportation', in Handbooks in Operations Research and Management Science, vol. 14, C. Barnhart and G. Laporte, Eds. Elsevier, 2007, pp. 467-537. https://doi.org/10.1016/S0927$\underline{0507(06) 14008-6 . b b}$

This article is an open access article distributed under the terms and conditions of the Creative Commons Attribution NonCommercial ( $C C B Y-N C$ 4.0) license. 